\title{
Putative Factors That May Modulate the Effect of Exercise on Liver Fat: Insights from Animal Studies
}

\author{
Faidon Magkos $1,2,3$ \\ ${ }^{1}$ Department of Nutrition and Dietetics, Harokopio University, 17671 Athens, Greece \\ ${ }^{2}$ Division of Endocrinology, Diabetes, and Metabolism, Beth Israel Deaconess Medical Center, Harvard Medical School, Boston, \\ MA 02215, USA \\ ${ }^{3}$ Department of Geriatrics \& Nutritional Science, Center for Human Nutrition, Washington University School of Medicine, \\ Campus Box 8031, Saint Louis, MO 63110, USA
}

Correspondence should be addressed to Faidon Magkos, faimag@hua.gr

Received 13 June 2011; Accepted 11 July 2011

Academic Editor: Jean-Marc Lavoie

Copyright () 2012 Faidon Magkos. This is an open access article distributed under the Creative Commons Attribution License, which permits unrestricted use, distribution, and reproduction in any medium, provided the original work is properly cited.

\begin{abstract}
An increase in intrahepatic triglyceride (IHTG) content is the hallmark of nonalcoholic fatty liver disease (NAFLD) and is strongly associated with insulin resistance and dyslipidemia. Although regular aerobic exercise improves metabolic function, its role in regulating fat accumulation in the liver is incompletely understood, and human data are scarce. Results from exercise training studies in animals highlight a number of potential factors that could possibly mediate the effect of exercise on liver fat, but none of them has been formally tested in man. The effect of exercise on IHTG content strongly depends on the background diet, so that exercise is more effective in reducing IHTG under conditions that favor liver fat accretion (e.g., when animals are fed high-fat diets). Concurrent loss of body weight or visceral fat does not appear to mediate the effect of exercise on IHTG, whereas sex (males versus females), prandial status (fasted versus fed), and duration of training, as well as the time elapsed from the last bout of exercise could all be affecting the observed exercise-induced changes in IHTG content. The potential importance of these factors remains obscure, thus providing a wide array of opportunities for future research on the effects of exercise (and diet) on liver fat accumulation.
\end{abstract}

\section{Introduction}

Excessive accumulation of fat in the liver, that is, intrahepatic triglyceride (IHTG), is associated with increased prevalence rates of and risk for dyslipidemia, diabetes, and cardiovascular disease [1-3]. Data from epidemiological as well as metabolic studies indicate that increased IHTG content is accompanied by insulin resistance and dysregulation of lipid metabolism [4-6]. Exercise is known to improve metabolic function $[7,8]$; however its effects on IHTG remain elusive $[9,10]$. Data from studies in humans are scarce and not entirely consistent [11]. In this paper, the results from a number of animal studies are briefly reviewed in an attempt to highlight putative factors that may modulate the effect of exercise on IHTG content.

\section{Exercise Training in Animals}

Many studies have evaluated the effect of aerobic exercise training on IHTG content in rodents; their design varies in terms of sex, strain, background diet, training duration, the prandial status, and the time of assessment after the last bout of exercise (Table 1) [12-37]. Results are largely heterogeneous, but a crude analysis of the data suggests that endurance training decreases IHTG (median: - 16\%, range: $-92 \%$ to $+97 \%, n=50$ studies; Table 1$)$. Most frequently $[14,16,18,20,26,31,35]$ but not always [15, 17, 34, 37], exercise has been shown to be more effective in reducing liver fat or attenuating its accretion in animals fed high-fat rather than standard, low-fat diets (median decrease: $25 \%$ and $14 \%$, resp., Figure 1(a)). This is consistent with data from human studies, in which exercise training appears to be more potent in reducing IHTG in subjects with increased baseline IHTG, for example, subjects with NAFLD, type II diabetes, or the elderly [11].

The reasons why exercise is more effective in reducing IHTG on high-fat than low-fat diets are not entirely clear but are likely related to the hepatosteatotic effect of high-fat feeding. Fat is mainly stored as microvesicles $\left(<1 \mu \mathrm{m}^{2}\right)$ within 


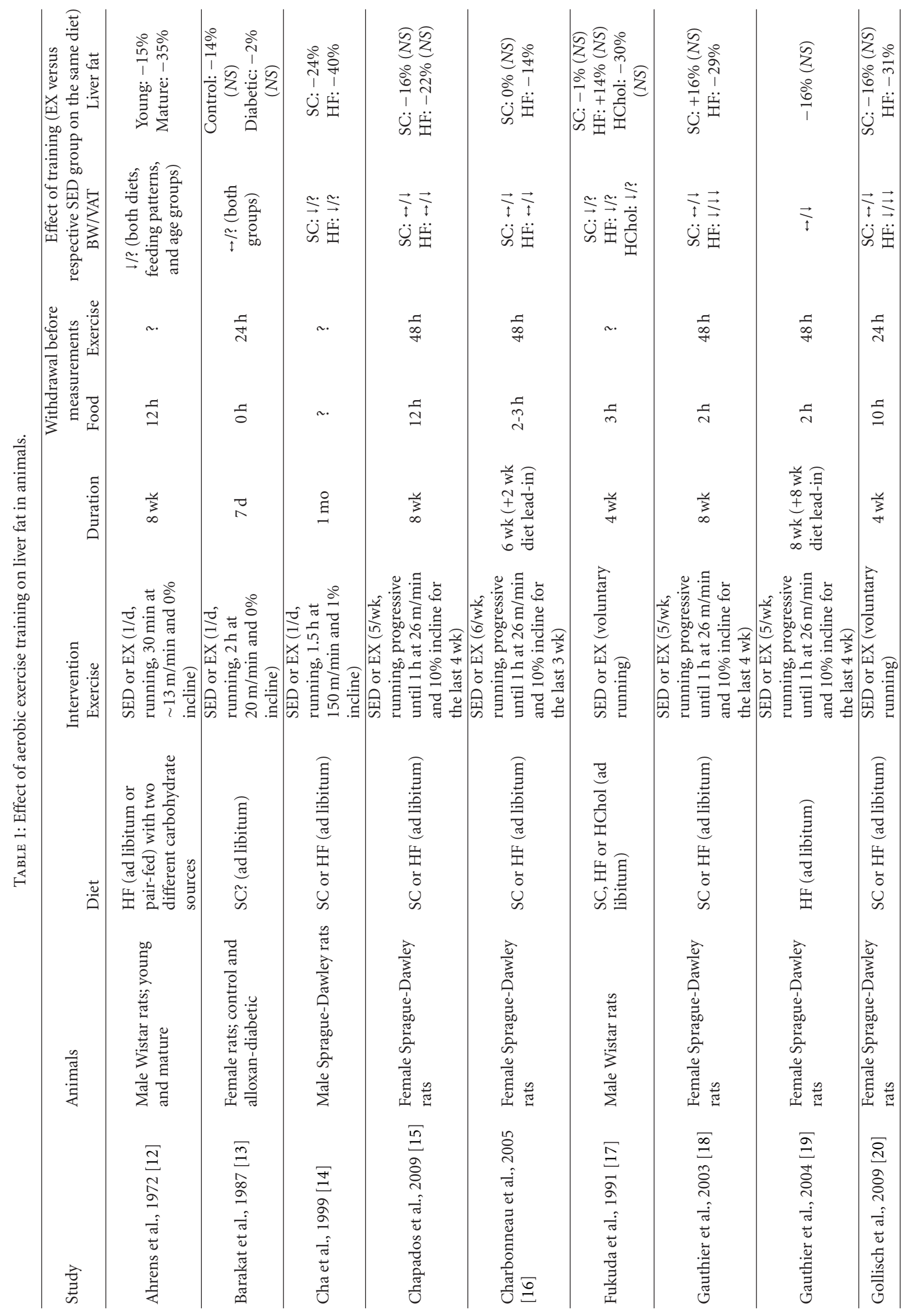




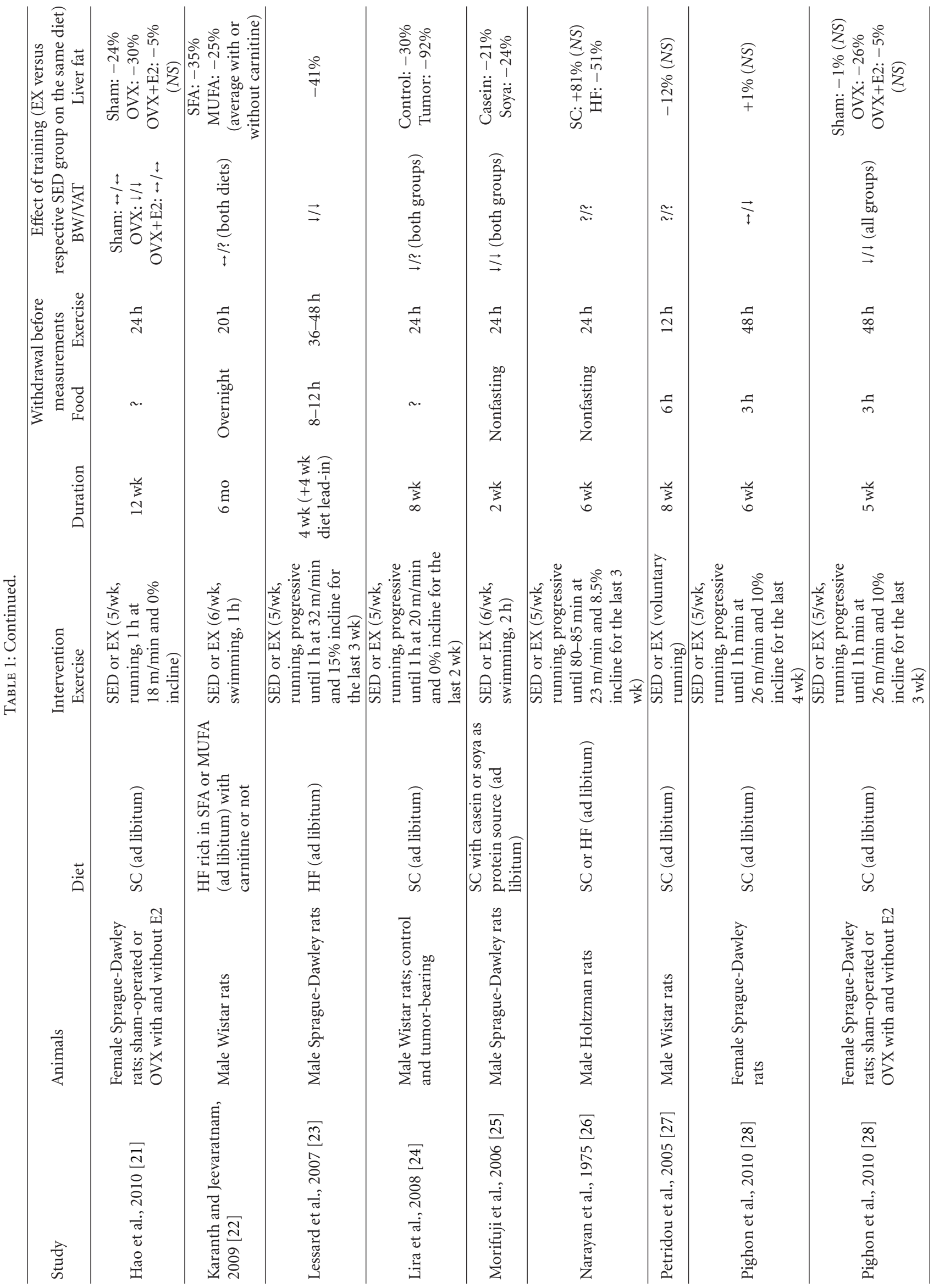




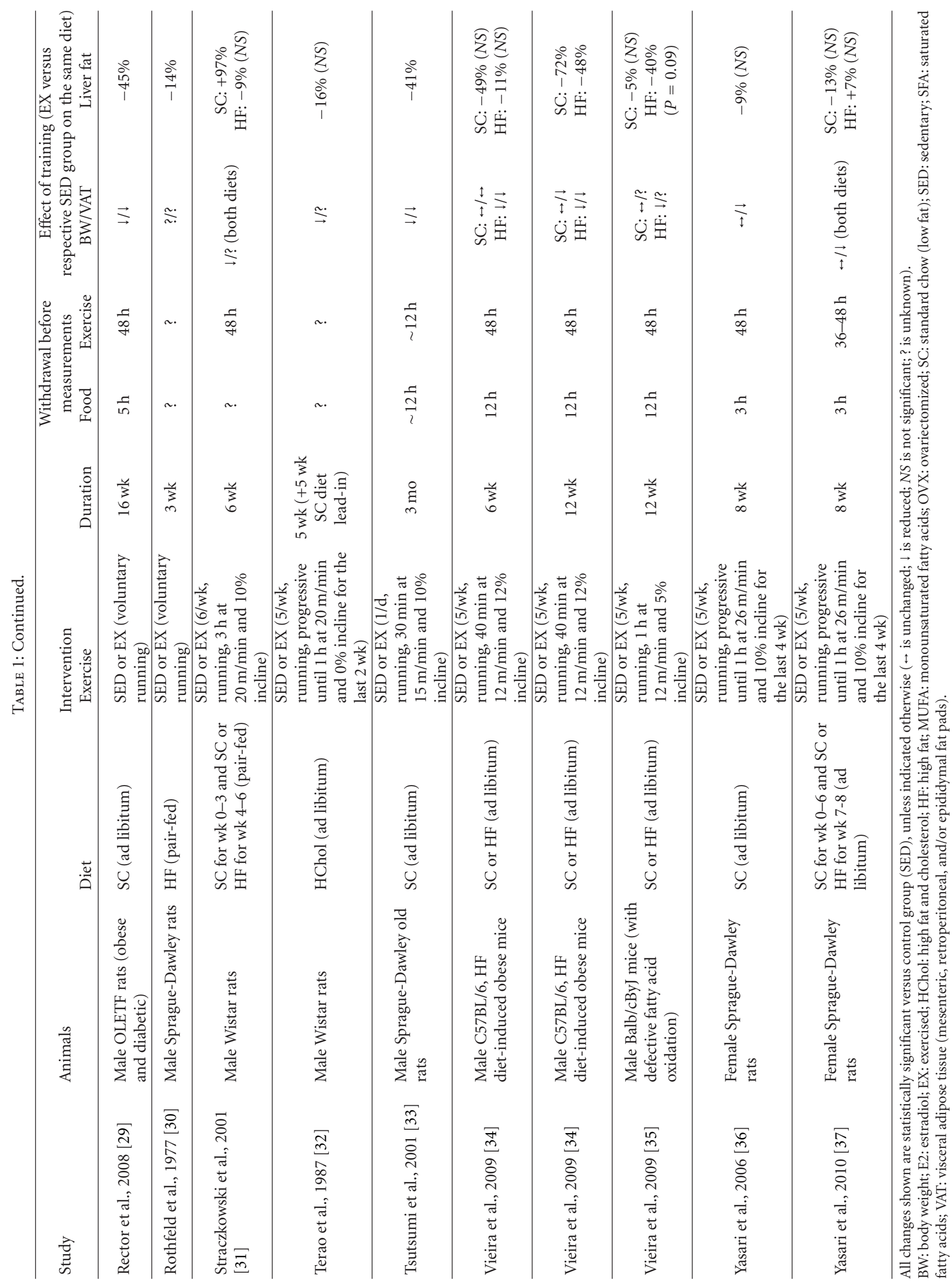




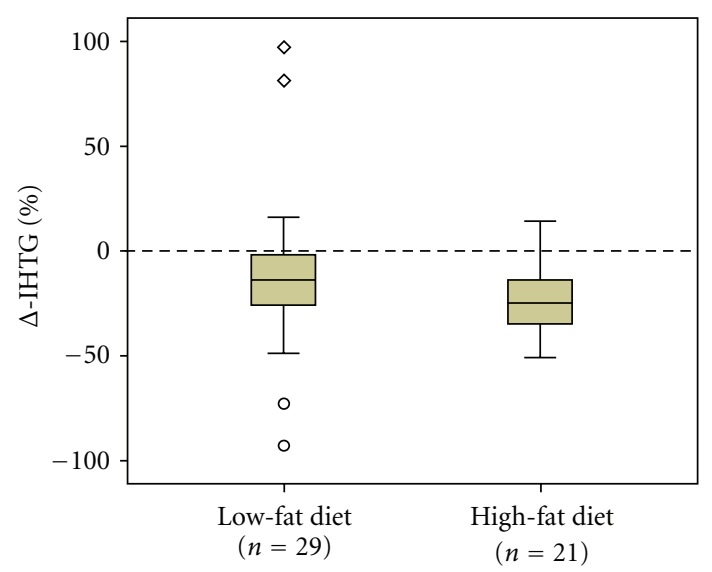

(a)

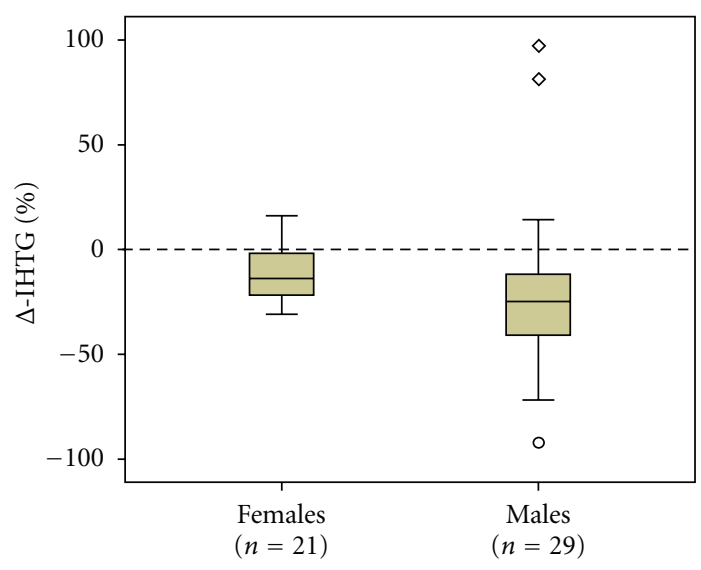

(c)

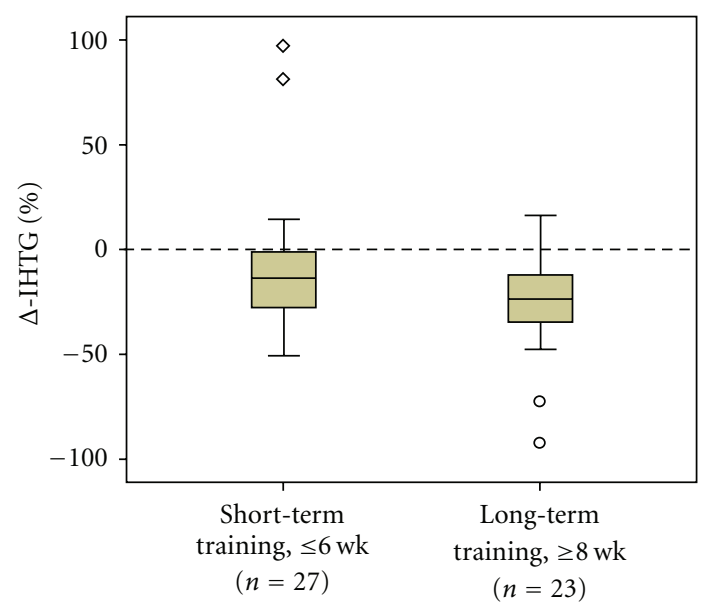

(e)

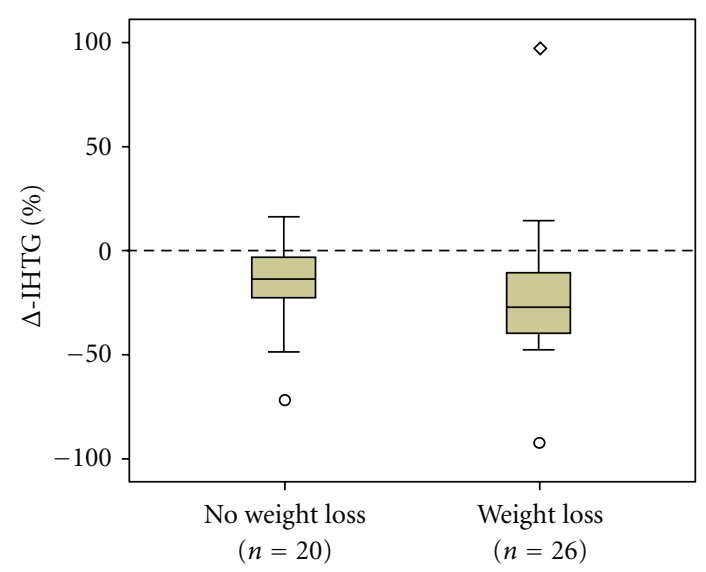

(b)

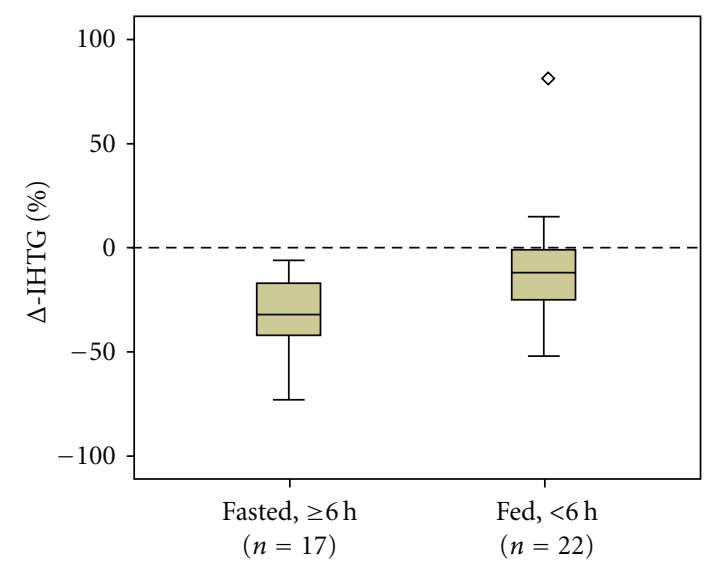

(d)

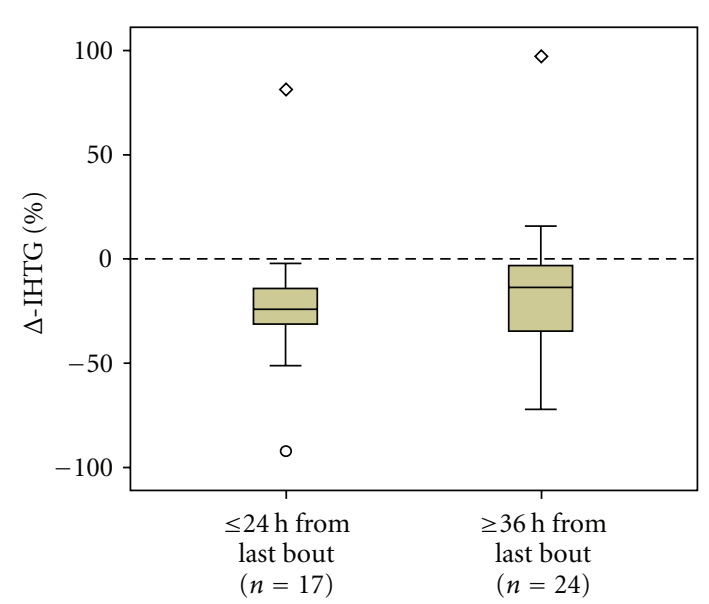

(f)

FIGURE 1: Factors that may affect changes in liver fat in response to exercise training in animals. Exercise-induced changes in intrahepatic triglyceride content $(\Delta$-IHTG) are shown for: (a) animals fed high fat or standard chow (low fat) diets; (b) animals that experienced weight loss (or attenuated weight gain) or not; (c) male or female animals; (d) fasted or fed animals; (e) animals trained for longer or shorter periods of time; (f) animals examined within one day from the last bout of exercise or later during recovery. Box plots have been constructed using average changes in liver fat (\% difference relative to sedentary controls) for each group of animals in the studies depicted in Table 1, and illustrate median, first, and third quartiles, minimum and maximum values, as well as potential positive and negative outliers. 
hepatocytes, whereas the high-fat diet-induced hepatic steatosis occurs via accumulation of macrovesicles $\left(>1 \mu \mathrm{m}^{2}\right)$ $[18,38]$. Endurance training has been shown to completely prevent the high-fat diet-induced hepatic steatosis [18, 38], that is, the hepatocyte surface area occupied by the lipid vacuoles, solely by reducing the number of lipid vacuoles in all sizes between 1 and $10 \mu \mathrm{m}^{2}$ (i.e., macrovesicles), without affecting the number of vacuoles of surface area $<1 \mu \mathrm{m}^{2}$ (i.e., microvesicles) [18]. Hence exercise may have less of an effect when on low-fat diets, not only because of lower total IHTG content, but also because most of this fat $(\sim 75 \%)$ is stored in microvesicles, not macrovesicles. A more pronounced IHTGdepleting effect of exercise has also been observed under other conditions that favor the development of fatty liver, such as overfeeding [34], ovariectomy [28], ethanol ingestion [39], or tumor-bearing [24]. Apart from the fat content of the background diet, the type of dietary carbohydrate [12], protein [25], and fat (i.e., saturated or unsaturated fatty acids) [22], as well as the feeding pattern (ad libitum or paired) [12] do not appear to affect, at least not in a major way, the exercise-induced change in IHTG content.

The collective of available data in animals highlights a number of other putative factors that may modulate the effect of exercise on liver fat; however, none of these factors has been formally tested using rigorous experimental designs. Concurrent weight loss or attenuated weight gain is not likely critical for the exercise-induced depletion of IHTG to manifest, albeit they may lead to greater reductions in liver fat when compared to no weight loss or similar weight gain (median decrease: $27.5 \%$ and 14\%, resp., Figure 1(b)). However, just like in humans [11], loss of visceral adipose tissue mass with exercise training is not necessarily coupled with a corresponding decrease in liver fat [16, 18, 28, 37]. Likewise, human studies have shown that exercise-induced reductions in IHTG content can occur in the absence of changes in total body fat [40] or even visceral adipose tissue [41].

Exercise may be more effective in reducing IHTG content in males than in females (median decrease: $25 \%$ and $14 \%$, resp., Figure $1(\mathrm{c}))$, in fasted $(\geq 6 \mathrm{~h})$ than in fed animals (median decrease: $31 \%$ and $11 \%$, resp., Figure $1(\mathrm{~d})$ ), and after longer ( $\geq 8 \mathrm{wk}$ ) than shorter interventions [34] (median decrease: $24 \%$ and 14\%, resp.; Figure 1(e)). The time elapsed from the last bout of exercise $(\leq 24 \mathrm{~h}$ or $\geq 36 \mathrm{~h})$ may also mediate the observed changes in IHTG (median decrease: $24 \%$ and $13.5 \%$, resp., Figure $1(\mathrm{f})$ ), suggesting that even acute exercise could affect liver fat. However, relevant information is scarce and inconclusive. A single bout of aerobic exercise (30-60 min) did not affect IHTG content, measured immediately after exercise, in female rats [42] but caused a $\sim 30 \%$ decrease in male rats [43] of the same strain, under both standard and high-fat feeding conditions. This is in line with data from exercise training studies in animals raising the possibility that males may be more sensitive to the IHTGreducing effect of exercise than females (Figure 1(c)), as well as with recent observations in humans [44]. Studies in which male rats were exercised until exhaustion provide conflicting results, some observed a mild [45] or marked $[46,47]$ increase in hepatic steatosis whereas others found a decrease of $30-60 \%$ [48] at the end of exercise.

\section{Detraining after Regular Exercise}

If regular exercise reduces liver fat, cessation of exercise should lead to an increase in IHTG content. Only a few animal studies have evaluated the effect of detraining on liver fat accumulation, and all have demonstrated that cessation of regular exercise (after 6-16 weeks of training) for a short (23 days) or a long ( 6 weeks) period of time is not associated with any significant changes in IHTG content compared with the trained state (i.e., before discontinuation of exercise) when animals are fed a standard low-fat diet $[28,36,49,50]$. Furthermore, detraining for 2-7 days does not alter the total number of hepatocyte lipid vacuoles and their size, even though it does activate precursors and processes in the liver known to initiate steatosis (e.g., decreased mitochondrial oxidative capacity, increased expression of de novo lipogenesis proteins, and increased malonyl CoA levels) [49]. Interpretation of detraining data is not straightforward, though. It is possible that this lack of an effect of detraining relates to the lesser potency of exercise in reducing liver fat content in animals fed standard low-fat diets (Figure 1), so that changes after detraining are similarly less pronounced. For instance, two $[28,36]$ out of three studies that reported no effect of detraining on liver fat also failed to observe a training-induced decrease in IHTG content, suggesting that training and detraining have no effect on liver fat accumulation under low-fat feeding conditions. Whereas one study [49] did observe a training-induced decrease in liver fat in rats fed a low-fat diet but found no changes after detraining, implying that the IHTG-depleting effect of regular exercise is long-lived and is not readily reversed by detraining. Still, compared with sedentary, never-exercised counterparts, detrained animals appear to be relatively protected from mild hepatic steatosis induced by 2 weeks of high-fat feeding [36], but not from the development of frank fatty liver 6 weeks after ovariectomy [28], even though cessation of exercise training in ovariectomized rats resulted in a nearly $40 \%$ increase in IHTG content compared with ovariectomized rats who did not stop exercising [28]. It is thus possible that the relevant molecular and biochemical adaptations to exercise are readily reversed when the exercise routine is interrupted $(<1$ week), however, changes in IHTG manifest later in time and only when strong triggering factors for liver fat accretion coexist, such as high-fat feeding or ovariectomy. Support for this notion is provided by an earlier study, where detraining resulted in a striking increase in IHTG content only when animals were subjected to starvation and refeeding [50].

\section{Conclusion}

The effect of exercise on IHTG content has recently attracted much scientific interest in light of the apparent detrimental metabolic effects of excessive liver fat accumulation. Although the results from a few studies in human subjects are promising, as exercise appears to reduce IHTG [11], the importance of the factors highlighted herein on the basis of studies in animals has never been evaluated in man. Future studies should at least control for-in order to avoid 
confounding — or directly investigate the role of these factors in affecting the exercise-induced changes in liver fat content.

\section{Conflict of Interests}

The author declares that there is no conflict of interests.

\section{References}

[1] L. A. Adams, J. F. Lymp, J. S. Sauver et al., "The natural history of nonalcoholic fatty liver disease: a population-based cohort study," Gastroenterology, vol. 129, no. 1, pp. 113-121, 2005.

[2] P. J. Hsiao, K. K. Kuo, S. J. Shin et al., "Significant correlations between severe fatty liver and risk factors for metabolic syndrome," Journal of Gastroenterology and Hepatology, vol. 22, no. 12, pp. 2118-2123, 2007.

[3] G. Targher and G. Arcaro, "Non-alcoholic fatty liver disease and increased risk of cardiovascular disease," Atherosclerosis, vol. 191, no. 2, pp. 235-240, 2007.

[4] E. Fabbrini, S. Sullivan, and S. Klein, "Obesity and nonalcoholic fatty liver disease: biochemical, metabolic, and clinical implications," Hepatology, vol. 51, no. 2, pp. 679-689, 2010.

[5] E. K. Speliotes, J. M. Massaro, U. Hoffmann et al., "Fatty liver is associated with dyslipidemia and dysglycemia independent of visceral fat: the Framingham heart study," Hepatology, vol. 51, no. 6, pp. 1979-1987, 2010.

[6] E. Fabbrini, F. Magkos, B. S. Mohammed et al., "Intrahepatic fat, not visceral fat, is linked with metabolic complications of obesity," Proceedings of the National Academy of Sciences of the United States of America, vol. 106, no. 36, pp. 15430-15435, 2009.

[7] F. Magkos, "Basal very low-density lipoprotein metabolism in response to exercise: mechanisms of hypotriacylglycerolemia," Progress in Lipid Research, vol. 48, no. 3-4, pp. 171-190, 2009.

[8] F. Magkos and L. S. Sidossis, "Exercise and insulin sensitivity: where do we stand? You'd better run!," European Journal of Endocrinology, vol. 4, no. 1, pp. 22-25, 2008.

[9] S. Caldwell and M. Lazo, "Is exercise an effective treatment for NASH? Knowns and unknowns," Annals of Hepatology, vol. 8, no. 1, pp. S60-S66, 2009.

[10] N. A. Spassiani and J. L. Kuk, "Exercise and the fatty liver," Applied Physiology, Nutrition and Metabolism, vol. 33, no. 4, pp. 802-807, 2008.

[11] F. Magkos, "Exercise and fat accumulation in the human liver," Current Opinion in Lipidology, vol. 21, no. 6, pp. 507-517, 2010.

[12] R. A. Ahrens, C. L. Bishop, and C. D. Berdanier, "Effect of age and dietary carbohydrate source on the responses of rats to forced exercise," Journal of Nutrition, vol. 102, no. 2, pp. 241247, 1972.

[13] H. A. Barakat, J. W. Carpenter, Y. A. Lennon, W. R. Hanna, K. F. O'Brien, and G. L. Dohm, "The effects of exercise on lipogenic enzyme activity and glyceride synthesis by liver homogenates of diabetic rats," Metabolism, vol. 36, no. 10, pp. 983-987, 1987.

[14] Y.-S. Cha, H.-S. Sohn, J. W. Daily III, and S.-H. Oh, "Effects of exercise training and/or high fat diet on lipid metabolism and carnitine concentrations in rats," Nutrition Research, vol. 19, no. 6, pp. 937-945, 1999.

[15] N. A. Chapados, M. Seelaender, E. Levy, and J. M. Lavoie, "Effects of exercise training on hepatic microsomal triglyceride transfer protein content in rats," Hormone and Metabolic Research, vol. 41, no. 4, pp. 287-293, 2009.
[16] A. Charbonneau, K. Couturier, M. S. Gauthier, and J. M. Lavoie, "Evidence of hepatic glucagon resistance associated with hepatic steatosis: reversal effect of training," International Journal of Sports Medicine, vol. 26, no. 6, pp. 432-441, 2005.

[17] N. Fukuda, M. Tojho, T. Hidaka, H. Sho, and M. Sugano, "Reciprocal responses to exercise in hepatic ketogenesis and lipid secretion in the rat," Annals of Nutrition and Metabolism, vol. 35, no. 4, pp. 233-241, 1991.

[18] M. S. Gauthier, K. Couturier, J. G. Latour, and J. M. Lavoie, "Concurrent exercise prevents high-fat-diet-induced macrovesicular hepatic steatosis," Journal of Applied Physiology, vol. 94, no. 6, pp. 2127-2134, 2003.

[19] M. S. Gauthier, K. Couturier, A. Charbonneau, and J. M. Lavoie, "ffects of introducing physical training in the course of a 16-week high-fat diet regimen on hepatic steatosis, adipose tissue fat accumulation, and plasma lipid profile," International Journal of Obesity and Related Metabolic Disorders, vol. 28, no. 8, pp. 1064-1071, 2004.

[20] K. S. Gollisch, J. Brandauer, N. Jessen et al., "Effects of exercise training on subcutaneous and visceral adipose tissue in normal- and high-fat diet-fed rats," American Journal of Physiology -Endocrinology and Metabolism, vol. 297, no. 2, pp. E495-E504, 2009.

[21] L. Hao, Y. Wang, Y. Duan, and S. Bu, "Effects of treadmill exercise training on liver fat accumulation and estrogen receptor alpha expression in intact and ovariectomized rats with or without estrogen replacement treatment," European Journal of Applied Physiology, vol. 109, no. 5, pp. 879-886, 2010.

[22] J. Karanth and K. Jeevaratnam, "Effect of dietary lipid, carnitine and exercise on lipid profile in rat blood, liver and muscle," Indian Journal of Experimental Biology, vol. 47, no. 9, pp. 748-753, 2009.

[23] S. J. Lessard, D. A. Rivas, Z. P. Chen et al., "Tissue-specific effects of rosiglitazone and exercise in the treatment of lipidinduced insulin resistance," Diabetes, vol. 56, no. 7, pp. 18561864, 2007.

[24] F. S. Lira, F. L. Tavares, A. S. Yamashita et al., "Effect of endurance training upon lipid metabolism in the liver of cachectic tumour-bearing rats," Cell Biochemistry and Function, vol. 26, no. 6, pp. 701-708, 2008.

[25] M. Morifuji, C. Sanbongi, and K. Sugiura, "Dietary soya protein intake and exercise training have an additive effect on skeletal muscle fatty acid oxidation enzyme activities and mRNA levels in rats," The British Journal of Nutrition, vol. 96, no. 3, pp. 469-475, 2006.

[26] K. A. Narayan, J. J. McMullen, and D. P. Butler, "Effect of exercise on tissue lipids and serum lipoproteins of rats fed two levels of fat," Journal of Nutrition, vol. 105, no. 5, pp. 581-587, 1975.

[27] A. Petridou, M. G. Nikolaidis, A. Matsakas, T. Schulz, H. Michna, and V. Mougios, "Effect of exercise training on the fatty acid composition of lipid classes in rat liver, skeletal muscle, and adipose tissue," European Journal of Applied Physiology, vol. 94, no. 1-2, pp. 84-92, 2005.

[28] A. Pighon, R. Barsalani, S. Yasari, D. Prud'Homme, and J. M. Lavoie, "Does exercise training prior to ovariectomy protect against liver and adipocyte fat accumulation in rats?" Climacteric, vol. 13, no. 3, pp. 238-248, 2010.

[29] R. S. Rector, J. P. Thyfault, R. T. Morris et al., "Daily exercise increases hepatic fatty acid oxidation and prevents steatosis in Otsuka Long-Evans Tokushima Fatty rats," American Journal of Physiology, vol. 294, no. 3, pp. G619-G626, 2008. 
[30] B. Rothfeld, A. Levine, and A. Varady, "The effect of exercise on lipid deposition in the rat," Biochemical Medicine, vol. 18, no. 2, pp. 206-209, 1977.

[31] M. Straczkowski, I. Kowalska, S. Dzienis-Straczkowska, M. Kinalski, J. Górski, and I. Kinalska, "The effect of exercise training on glucose tolerance and skeletal muscle triacylglycerol content in rats fed with a high-fat diet," Diabetes and Metabolism, vol. 27, no. 1, pp. 19-23, 2001.

[32] T. Terao, T. Fujise, and S. Nakano, "Effects of long-term exercise and high-cholesterol diet on lipid-lipoprotein metabolism in rats," Tokai Journal of Experimental and Clinical Medicine, vol. 12, no. 4, pp. 243-251, 1987.

[33] K. Tsutsumi, M. Kusunoki, T. Hara et al., "Exercise improved accumulation of visceral fat and simultaneously impaired endothelium-dependent relaxation in old rats," Biological and Pharmaceutical Bulletin, vol. 24, no. 1, pp. 88-91, 2001.

[34] V. J. Vieira, R. J. Valentine, K. R. Wilund, N. Antao, T. Baynard, and J. A. Woods, "Effects of exercise and low-fat diet on adipose tissue inflammation and metabolic complications in obese mice," American Journal of Physiology-Endocrinology and Metabolism, vol. 296, no. 5, pp. E1164-E1171, 2009.

[35] V. J. Vieira, R. J. Valentine, K. R. Wilund, and J. A. Woods, "Effects of diet and exercise on metabolic disturbances in highfat diet-fed mice," Cytokine, vol. 46, no. 3, pp. 339-345, 2009.

[36] S. Yasari, A. Paquette, A. Charbonneau, M. S. Gauthier, R. Savard, and J. M. Lavoie, "Effects of ingesting a high-fat diet upon exercise-training cessation on fat accretion in the liver and adipose tissue of rats," Applied Physiology, Nutrition and Metabolism, vol. 31, no. 4, pp. 367-375, 2006.

[37] S. Yasari, D. Prud'Homme, D. Wang et al., "Exercise training decreases hepatic SCD-1 gene expression and protein content in rats," Molecular and Cellular Biochemistry, vol. 335, no. 1-2, pp. 291-299, 2010.

[38] Y. He, H. Zhang, and F. H. Fu, "The effects of swimming exercise on high-fat-diet-induced steatohepatitis," Journal of Sports Medicine and Physical Fitness, vol. 48, no. 2, pp. 259265, 2008.

[39] J. R. Trudell, W. Q. Lin, D. A. Chrystof, G. Kirshenbaum, and C. M. Ardies, "Induction of HSP72 in rat liver by chronic ethanol consumption combined with exercise: association with the prevention of ethanol-induced fatty liver by exercise," Alcoholism: Clinical and Experimental Research, vol. 19, no. 3, pp. 753-758, 1995.

[40] F. M. Finucane, S. J. Sharp, L. R. Purslow et al., "The effects of aerobic exercise on metabolic risk, insulin sensitivity and intrahepatic lipid in healthy older people from the Hertfordshire Cohort Study: a randomised controlled trial," Diabetologia, vol. 53, no. 4, pp. 624-631, 2010.

[41] S. Bonekamp, B. B. Barone, J. Clark, and K. J. Stewart, "The effect of an exercise training intervention on hepatic steatosis," Hepatology, vol. 48, supplement 1, p. 806A, 2008.

[42] A. Charbonneau, A. Melancon, C. Lavoie, and J. M. Lavoie, "Alterations in hepatic glucagon receptor density and in Gs $\alpha$ and Gi $\alpha 2$ protein content with diet-induced hepatic steatosis: effects of acute exercise," American Journal of PhysiologyEndocrinology and Metabolism, vol. 289, no. 1, pp. E8-E14, 2005.

[43] A. Charbonneau, C. G. Unson, and J. M. Lavoie, "Highfat diet-induced hepatic steatosis reduces glucagon receptor content in rat hepatocytes: potential interaction with acute exercise," Journal of Physiology, vol. 579, no. 1, pp. 255-267, 2007.

[44] S. Haufe, S. Engeli, P. Budziarek et al., "Cardiorespiratory fitness and insulin sensitivity in overweight or obese subjects may be linked through intrahepatic lipid content," Diabetes, vol. 59, no. 7, pp. 1640-1647, 2010.

[45] H. M. Maling, D. N. Stern, P. D. Altland, B. Highman, and B. B. Brodie, "The physiologic role of the sympathetic nervous system in exercise," Journal of Pharmacology and Experimental Therapeutics, vol. 154, no. 1, pp. 35-45, 1966.

[46] J. Gorski and T. Kiryluk, "The post-exercise recovery of triglycerides in rat tissues," European Journal of Applied Physiology and Occupational Physiology, vol. 45, no. 1, pp. 3341, 1980.

[47] P. D. Altland and B. Highman, "Effects of exercise on serum enzyme values and tissues of rats," American Journal of Physiology, vol. 201, pp. 393-395, 1961.

[48] M. Straczkowski, I. Kowalska, J. Górski, and I. Kinalska, "The effect of a single bout of exhaustive exercise on muscle carbohydrate and lipid metabolism in a rat model of type 2 diabetes mellitus," Acta Diabetologica, vol. 37, no. 1, pp. 4753, 2000 .

[49] S. R. Rector, J. P. Thyfault, M. J. Laye et al., "Cessation of daily exercise dramatically alters precursors of hepatic steatosis in Otsuka Long-Evans Tokushima Fatty (OLETF) rats," Journal of Physiology, vol. 586, no. 17, pp. 4241-4249, 2008.

[50] P. Lowney, V. M. Lee, R. J. Hansen, and J. S. Stern, "Effects of exercise, detraining, starvation, and refeeding on lipogenic capacity of Osborne-Mendel rat," American Journal of Physiology, vol. 254, no. 4, pp. R648-R654, 1988. 


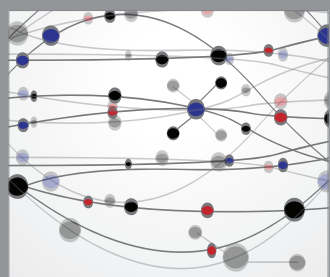

The Scientific World Journal
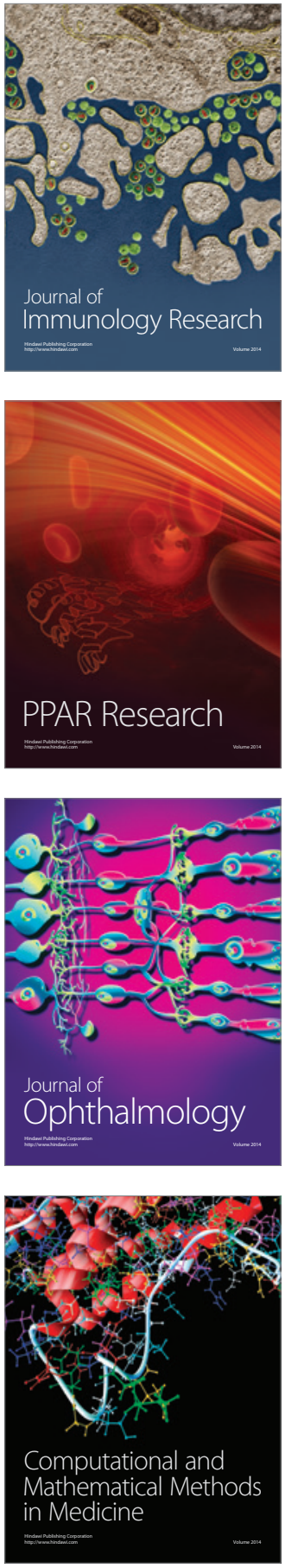

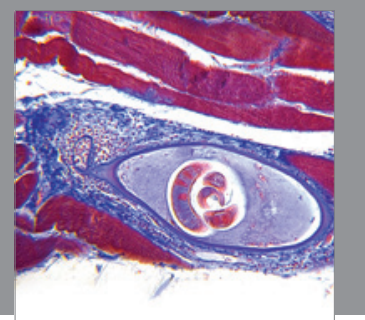

Gastroenterology

Research and Practice
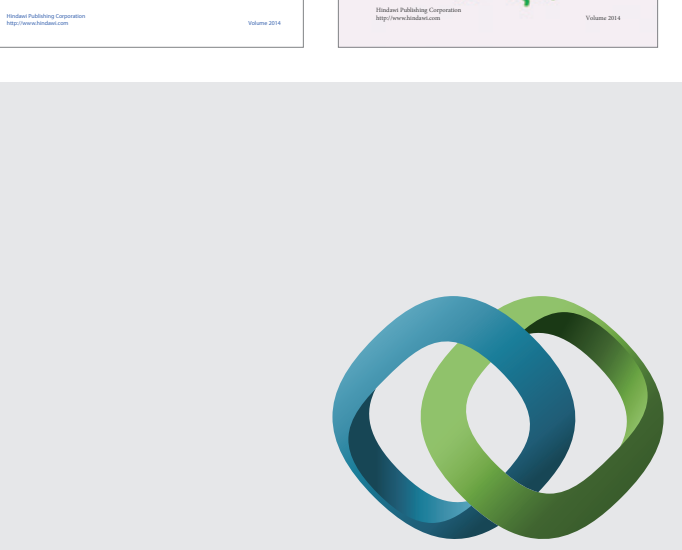

\section{Hindawi}

Submit your manuscripts at

http://www.hindawi.com
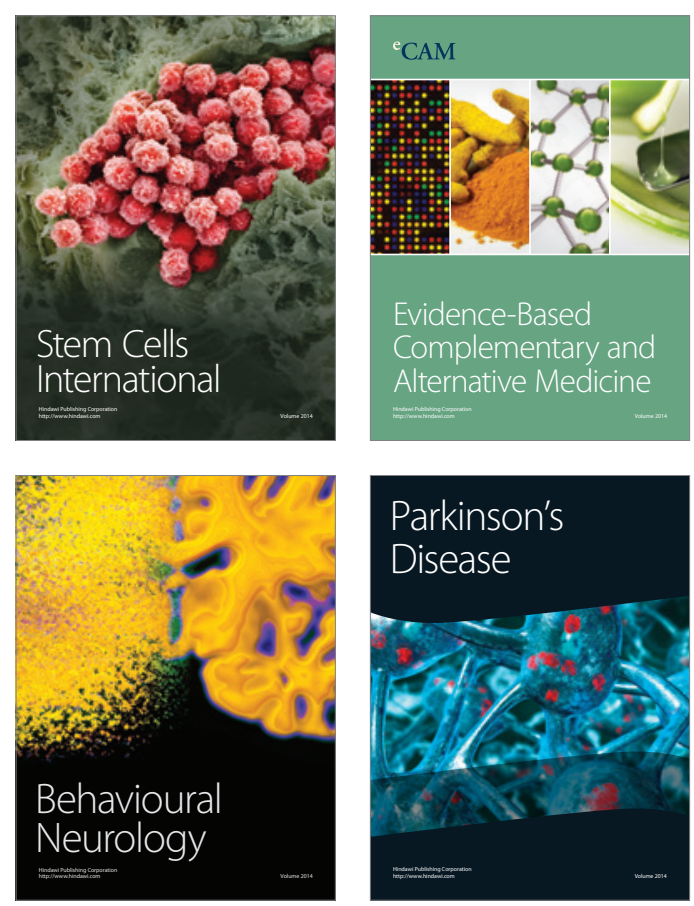

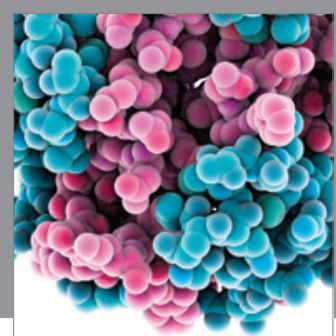

Journal of
Diabetes Research

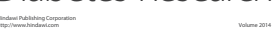

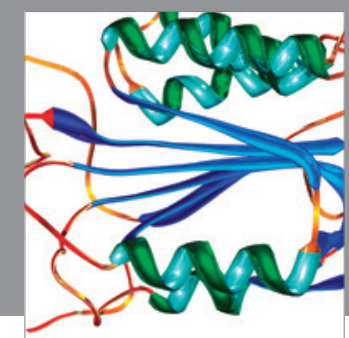

Disease Markers
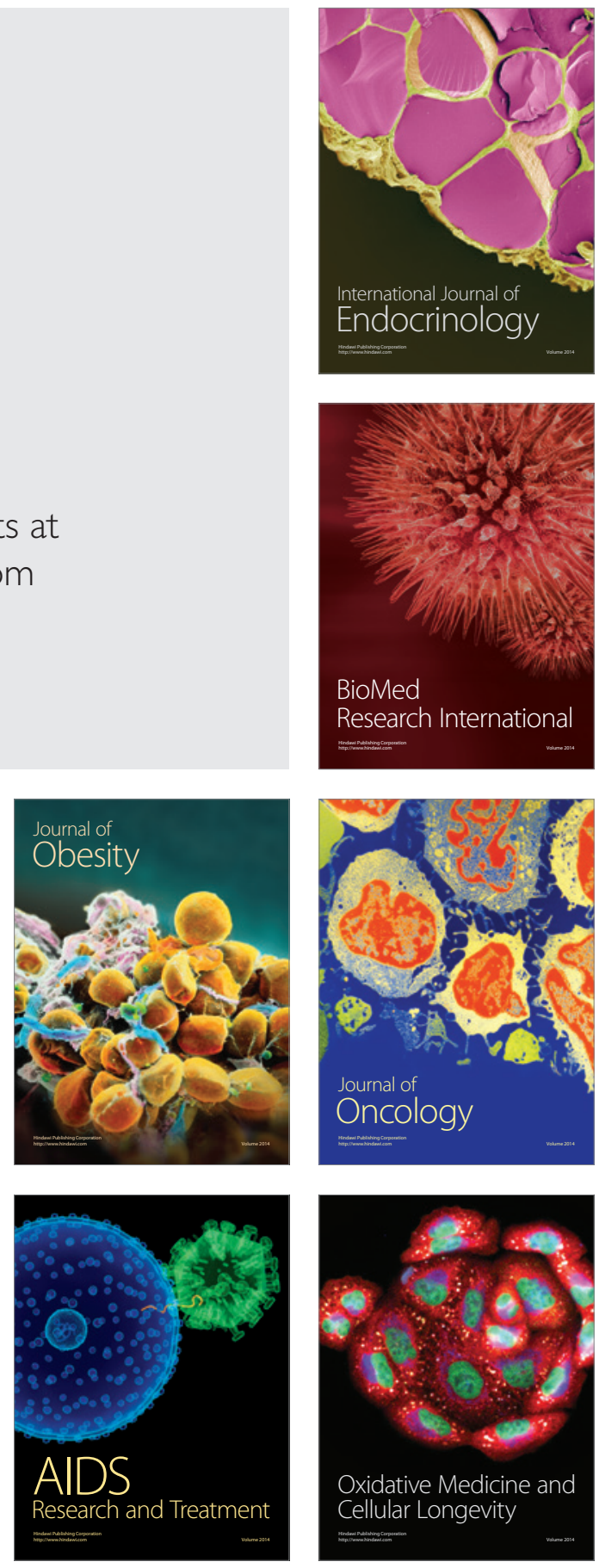